\title{
Unveiling of Dominant Fungal Pathogens Associated With Rusty Root Rot of Panax notoginseng Based on Multiple Methods
}

Chunyi Mi and Ruihuan Yang, Zhejiang Province Key Laboratory of Plant Secondary Metabolism and Regulation, College of Life Science, Zhejiang Sci-Tech University, 310018, Hangzhou; Junfeng Rao, Hangzhou Vocational \& Technical College, 310018, Hangzhou; Shaozhou Yang and Fugang Wei, Sanqi Technology Co. Ltd., 663000, Wenshan City, P. R. China; and Ou Li ${ }^{\dagger}$ and Xiufang Hu, Zhejiang Province Key Laboratory of Plant Secondary Metabolism and Regulation, College of Life Science, Zhejiang Sci-Tech University, 310018, Hangzhou

\begin{abstract}
Root rot is an important disease hampering the sustainable cultivation of Panax notoginseng. Culture-dependent and independent techniques were used to elucidate the dominant fungal pathogen of rusty root rot of $P$. notoginseng. Based on Illumina sequencing profiles for fungi using ITS primers, five phyla - namely Ascomycota, Basidiomycota, Glomeromycota, Zygomycota, and Chytridiomycota - were identified, and the analyses showed that the Ascomycota was the dominant phylum ( $\sim 50$ to $97 \%$ ), especially in the symptomatic samples. Out of 226 total genera identified, seven genera had over $1 \%$ average abundance, including Ilyonectria, Fusarium, Tetracladium, Cladosporium, Rhizophagus, Alternaria,

and Perisporiopsis. However, only Ilyonectria was the predominant genera in the symptomatic samples ( $\sim 76$ to $80 \%)$, while the others, including Fusarium, had higher abundances in asymptomatic samples. Based on in vitro and in vivo pathogenicity, the isolate G3B was demonstrated to be the pathogen causing rusty root rot of $P$. notoginseng, and it was identified as Ilyonectria mors-panacis. Based on primers F2-R2 targeting the His3 gene of Ilyonectria, real-time quantitative PCR (qPCR) was performed as an additional proof confirming that $I$. mors-panacis was the dominant pathogen in the symptomatic samples during the years of the study (2014-2015).
\end{abstract}

Panax notoginseng (Burk.) F. H. Chen, of the family Araliaceae, is a traditional Chinese medicinal plant (Hong et al. 2014). Notoginsenoside $\mathrm{R} 1$ and ginsenosides $\mathrm{Rg} 1, \mathrm{Rd}, \mathrm{Re}$, and $\mathrm{Rb} 1$ are its principal active constituents, which endow a wide range of pharmacological activities, such as anti-inflammatory, antifatigue, and hepato-protection effects, and coronary heart disease prevention ( $\mathrm{Li}$ et al. 2007; Xia et al. 2016). This plant has been cultivated mainly in the southwest regions of China, especially in the Wenshan region of Yunnan Province, for more than 400 years (Ma et al. 2013).

Panax notoginseng is seeded in the fall for emergence the following spring, and the roots require 3 years of growth prior to harvest (Guo et al. 2010). It prefers a warm, wet, shady, and cool environment, with soil humidity ranging from 25 to $30 \%$ and relative air humidity from 70 to $85 \%$ (Guan et al. 2011), conditions which also facilitate the infection of numerous phytopathogens (Chen et al. 2001). Root rot is the most destructive disease of $P$. notoginseng, which significantly reduces root quality and decreases yield by 5 to $20 \%$, sometimes even exceeding 70\% (Sun et al. 2004; Wu et al. 2015). Root rot has been the major threat to cultivation and sustainable development of this plant. Clarification of the dominant pathogens is therefore a prerequisite for effective control of the disease, which is vital for the continuous development of $P$. notoginseng.

In fact, root rot is a ubiquitous disease worldwide, occurring in multiple plants with varied pathogens. Root rot has been reported to occur in many crops (Scherm et al. 2013), fruits (Reis et al. 2013; Tewoldemedhin et al. 2011), vegetables (Shishido 2014), and medicinal plants (Latha et al. 2011). Root rot induces severe damage and high reduction in yields worldwide (Seifert et al. 2003). The reported pathogens associated with root rot include bacteria such as Pseudomonas spp., Pectobacterium carotovorum, and Ralstonia spp. (Heydari et al. 2014; Hu et al. 2008),

${ }^{\dagger}$ Corresponding authors: O. Li; E-mail: ouli@zstu.edu.cn and X. Hu; E-mail: huxiuf@zstu.edu.cn

*The $\boldsymbol{e}$-Xtra logo stands for "electronic extra" and indicates that one supplementary table and three supplementary figures are published online.

Accepted for publication 9 July 2017.

@ 2017 The American Phytopathological Society and fungi such as Alternaria spp., Cylindrocarpon spp., Fusarium spp., Phytophthora cactorum, Phoma herbarum, Lasiodiplodia theobromae, and Rhizoctonia solani (Abdel-Monaim and Abo-Elyousr 2012; Latha et al. 2011; Miao et al. 2006). Cylindrocarpon destructans and Ilyonectria robusta were reported to cause rusty root rot of $P$. ginseng in China and other nations (Cabral et al. 2012; Lu et al. 2015).

Root rot is a worsening problem for P. notoginseng in Wenshan, China (Miao et al. 2006). It is usually a typical rusty rot, with characteristic brown, sunken, necrotic lesions on the roots, and chlorotic leaves. Until now, the reported pathogens associated with $P$. notoginseng included Cylindrocarpon destructans, $C$. didynum, Fusarium solani, Phytophthora cactorum, Phoma herbarum, Monilia sp., Verticillium sp., Trichoderma sp., and Rhizoctonia solani (Mao et al. 2014; Miao et al. 2006). However, confusion remains as to the exact identity of the dominant pathogens causing root rot.

Culture-independent methods are increasingly being used to investigate the profiles of microbial communities. Culture-dependent methods are valuable for studying several microorganisms but it is unfortunately impossible to use these methods for the identification of all of them. Recently, rapid developments in molecular microbial ecology have allowed culture-dependent methods to be complemented by the collection of unprecedented quantities of 16S rRNA or ITS data (Caporaso et al. 2011). Molecular methods, such as terminal-restriction fragment length polymorphism (T-RFLP), denaturing and temperature gradient gel electrophoresis (DGGE/TGGE), and novel barcoded pyrosequencing, are becoming widely used in the studies of communities of microorganisms (Cardenas and Tiedje, 2008; Tringe and Hugenholtz, 2008). All these methods have been employed successfully to investigate microbial community diversity in environments (Remington et al. 2001) and hosts (Yu et al. 2013).

The aim of this study is to investigate the fungal communities and to determine the dominant pathogens causing rusty root rot of $P$. notoginseng based on culture-independent and dependent methods. The elucidation of the dominant pathogens will certainly help determine their roles in a disease that greatly impede sustainable cultivation of $P$. notoginseng and ultimately help provide solutions to this problem.

\section{Materials and Methods}

Sampling. Panax notoginseng plants were grown by Sanqi Technology Co. Ltd. $\left(23^{\circ} 53^{\prime} \mathrm{N}, 104^{\circ} 23^{\prime} \mathrm{E}\right)$ in Miaoxiang, Wenshan City, 
Yunnan Province, China. The area has a subtropical and continental monsoon climate, with an annual average temperature of $16^{\circ} \mathrm{C}$ and an average annual precipitation of $1200 \mathrm{~mm}$. In 2014, both asymptomatic and symptomatic plants were sampled from two different sites (2014S1 and 2014S2). In 2015, the samples were separately collected from 2- and 3-year-old planting sites (2015S1DR2 and 2015S2DR3) per month during May and September. Altogether, six batches of samples were obtained from the two sites, with each batch consisting of six plants (asymptomatic and symptomatic, Supplementary Fig. S1) from each site. All the plants were transported to the laboratory for sterilization and further experiments on the same day. For sterilization, the samples were rinsed under running water for $30 \mathrm{~min}$, immersed in $70 \%$ ethanol for $30 \mathrm{~s}$ and then in $1 \%$ sodium hypochlorite for $3 \mathrm{~min}$, and washed with $\mathrm{ddH}_{2} \mathrm{O}$.

DNA extraction. Total genomic DNA was extracted from $1 \mathrm{~g}$ of sterilized root material for each sample using the modified cetyltrimethyl ammonium bromide (CTAB) method (Yu et al. 2013), and the quality of genomic DNA was determined by agarose gel electrophoresis and quantified by means of a NanoDrop ND-1000 Spectrophotometer.

Analysis of fungal communities. Amplicon library construction and Illumina sequencing. Illumina sequencing of the Internal Transcribed Spacer 2 (ITS2) was performed on four samples, including asymptomatic (plant root, PLR) and symptomatic (plant diseased root, PLDR) roots from 2- and 3-year-old (Y2 and Y3) planting sites. The ITS2 region was amplified from root samples using primers ITS906 (5'-AACTTTYRRCAAYGGATCTC-3') and 1453R (5'AAGTTCAGCGGGTADY CCTA-3') (Qian et al. 2016). The reaction mixture included $5 \mu \mathrm{l}$ of $10 \times$ PCR buffer, $0.5 \mu l$ of $10 \mathrm{mM}$ dNTPs, $0.5 \mu l$ of $50 \mathrm{mM}$ each primer, $0.5 \mu \mathrm{l}$ of Taq polymerase, genomic DNA $(10 \mathrm{ng})$, and $\mathrm{dd}_{2} \mathrm{O}$ added to achieve a final volume of $50 \mu \mathrm{l}$. The reactions were carried out under the following conditions: $94^{\circ} \mathrm{C}$ for $3 \mathrm{~min} ; 5$ cycles of $94^{\circ} \mathrm{C}$ for $30 \mathrm{~s}, 45^{\circ} \mathrm{C}$ for $20 \mathrm{~s}$, and $65^{\circ} \mathrm{C}$ for $30 \mathrm{~s}$, followed by 20 cycles of $94^{\circ} \mathrm{C}$ for $20 \mathrm{~s}, 55^{\circ} \mathrm{C}$ for $20 \mathrm{~s}$, and $72^{\circ} \mathrm{C}$ for $30 \mathrm{~s}$; and finally, $72^{\circ} \mathrm{C}$ for $5 \mathrm{~min}$. Electrophoresis was performed to confirm successful amplification, and PCR products were purified from the agarose gel using a DNA gel extraction kit (Sangon Biotech Co. Ltd., Shanghai, China). The barcode-tagged fragments were then quantified using a Qubit dsDNA HS Assay kit with a Qubit2.0 fluorometer (Life Technologies, Grand Island, NY). Finally, equal amounts of amplicon for each sample were combined to obtain an equimolar pool, which was sequenced using $2 \times 300$ paired-end Illumina Miseq at the Shanghai Sangon Biotech Co. Ltd. (Shanghai, China).

Bioinformatics analyses. The attainment of raw sequencing data was accomplished by removing those sequences whose lengths did not meet the minimum quality filter criteria (200 bp), and by removing the barcode sequences and the joint sequences, thus ensuring the acquisition of high-quality and credible data. Unique sequences were then aligned against SILVA and chimeric sequences were removed using UCHIME. The effective sequences of each sample were clustered using UCLUST v1.1.579 to build operational taxonomic unit (OTUs) with a cutoff value of $97 \%$ sequence identity.

Diversity indices were calculated using SPADE software (Hong et al. 2006). The RDP Classifier of the Ribosomal Database Project (RDP) and UNITE were used for fungal OTU representative sequences species classification analysis at a confidence threshold of 80\% (Hong et al. 2006). Taxonomic assignments from phylum level to genus level were then performed. The rarefaction curves and Alpha diversity were analyzed using mothur, including chao1, ACE values, Shannon indices, and coverage values (Schloss et al. 2009). Chao1, ACE, and Shannon index are, respectively, the total number of species, common indices of the estimated total species, and diversity index. For beta diversity analysis, principal component analysis (PCA) was conducted based on RDP Classifier taxa, the OTUs described above, and weighted Unifrac.

Isolation of fungi and their pathogenesis. Isolation of fungi was performed by transferring fragments of symptomatic roots of $P$. notoginseng, which were surface-sterilized, ground with a mortar and pestle, and diluted with $\mathrm{ddH}_{2} \mathrm{O}$. A series of sample dilutions $\left(10^{-1}\right.$, $10^{-2}, 10^{-3}, 10^{-4}$, and $10^{-5}$ ) were spread on potato dextrose agar (PDA) amended with streptomycin sulfate at $100 \mathrm{mg} / \mathrm{liter}$, with three repetitions per dilution, and then incubated at $25^{\circ} \mathrm{C}$ in the dark. After incubation for 14 days, morphologically different colonies were selected as candidate fungi and purified for storage and further experiments.

In vitro and in vivo tests were carried out on root cuttings and plants to determine the pathogenicity of the candidate fungi. The candidates were activated on PDA medium and cultured at $25^{\circ} \mathrm{C}$ in darkness for 7 to 14 days. The spores were washed with $\mathrm{dd}_{2} \mathrm{O}$ and made into a $10^{7} \mathrm{cfu} / \mathrm{ml}$ suspension. The asymptomatic roots of $P$. notoginseng were sterilized as above, and were cut into 5-mm slices and put on plates, with three slices per plate. Each slice was inoculated with 20- $\mu$ l spore suspension, and with $\mathrm{ddH}_{2} \mathrm{O}$ as the negative control. There were three repetitions for each treatment. Plates were kept in a $25^{\circ} \mathrm{C}$ incubator and routinely observed.

The pathogenicities of the candidates with symptoms on the root cuttings were further evaluated on 2-year-old potted plants of $P$. notoginseng. Trials were conducted on 10 plants per treatment which were inoculated with spore suspensions as above. The same number of plants, inoculated with $\mathrm{ddH}_{2} \mathrm{O}$, was used as a control. The potted plants were incubated in the laboratory at $25 \pm 2{ }^{\circ} \mathrm{C}$ for 4 weeks and were watered every 3 days. All the plants were observed daily, and the symptomatic roots were used for isolation to confirm the presence of the candidate fungi.

The pathogens found were characterized for phenotype and phylogeny. Phenotypic characteristics were examined on PDA or OA (1000 $\mathrm{ml}$ of juice from $200 \mathrm{~g}$ of oatmeal boiled for $15 \mathrm{~min}$ was solidified with $18 \mathrm{~g}$ of agar, including $0.1 \mathrm{~g}$ of $\mathrm{FeSO}_{4}, 0.1 \mathrm{~g}$ of $\mathrm{MnCl}_{2}$, and $0.1 \mathrm{~g}$ of $\mathrm{ZnSO}_{4}$ ) after cultivation at $25^{\circ} \mathrm{C}$ for 12 days in darkness. Genomic DNA extraction, amplification and sequencing of the ITS gene based on primers ITS1 (5'-TCCGTAGGTGAACCTGCGG$\left.3^{\prime}\right)$ and ITS4 (5'-TCCTCCGCTTATTGATATGC-3') were performed as described previously (Crous et al. 2006). For the dominant pathogen, the Histone gene (His3) was also amplified and sequenced using universal primers $\mathrm{H} 3$-1a (5'-ACTAAGCAGACCGCCCG CAGG-3') and H3-1b (5'-GCGGGCGAGCTGGATGTCCTT-3') (Glass and Donaldson 1995). The sequences were aligned with representatives from the related species that had been deposited in the GenBank, EMBL and DDBJ databases. Phylogenetic trees were constructed by neighbor-joining (MEGA version 3.1). Bootstrap resampling analysis was performed to estimate the confidence of the tree topologies.

Confirmation of the dominant pathogen based on qPCR. To confirm the dominant fungal pathogen in rusty root rot of $P$. notoginseng, symptomatic samples obtained during 2014-2015 were tested for fungal abundance using qPCR. Firstly, specific primers were designed according to the His 3 gene sequences corresponding to the species and genera of the dominant pathogens available in GenBank. Primers with specificity to the species were selected and synthesized by GENEWIZ Biological Technology Company (Suzhou, China). The specificity of the primers was evaluated through sequencing of the amplicon from the rotted roots of $P$. notoginseng.

The qualified primer pair, as well as universal primers NS1 and NS8 targeting the fungal 18S rRNA gene, were used for qPCR to determine the distribution of the dominant pathogen in $P$. notoginseng. The qPCR was conducted in an ABI 7500 system using SYBR Green Mater Mix (TaKaRa, Dalian, China) according to the instructions. All reactions were performed in triplicate, with $\mathrm{ddH}_{2} \mathrm{O}$ as the blank control. The threshold cycle $\left(\mathrm{C}_{\mathrm{T}}\right)$ values were determined automatically using an Applied Biosystem 7500 Sequence Detection System, and the relative ratios of the pathogens were calculated as relative quantity values using the comparative $C_{T}\left(2^{-\Delta \Delta C T}\right)$ method. Differences in values were examined using the $t$ test, and $P$ values of $<0.01$ were considered as significant.

\section{Results}

Differences in fungal communities between symptomatic and asymptomatic roots. Based on the Illumina sequencing data, a total of $63,920(97.40 \%$ of the raw 65,628$)$ high-quality ITS sequences passed the quality control criteria and the number of reads per sample ranged from 11,062 to 21,833 (Supplementary Table S1). OTUs of 
all the asymptomatic samples were higher than those of the symptomatic samples, which was in accordance with the Shannon index values. The highest OTUs (295) and Shannon index (2.75) values were observed in PLRY3 and PLRY2, respectively (Table 1), while the lowest OTUs and Shannon index values were obtained for PLDRY3. The richness rarefaction curves of the four samples are shown in Supplementary Figure S2. All the amplified rarefaction curves increased rapidly from 0 to 5,000 sequences, indicating that the sequence-derived diversity and richness in this study were sufficient to characterize the species in each sample.

Across the four sample types, we detected a total of five fungal phyla, namely Ascomycota, Basidiomycota, Glomeromycota, Zygomycota, and Chytridiomycota (Fig. 1). Ascomycota was the most dominant phylum, accounting for about 50.38 to $97.49 \%$ of total fungal ITS reads and more abundant in the symptomatic samples than in the asymptomatic ones, while it was the contrary for the Glomeromycota $(0.05$ to $0.11 \%$ ), which were much less abundant in the symptomatic samples. As for Zygomycota and Chytridiomycota (this group not shown in Fig. 1), both existed only in the symptomatic samples with abundance of 0.22 to $0.24 \%$ and $0.01 \%$, respectively.

At the genus level, 226 were identified from all the samples, and each of seven genera had over $1 \%$ average abundance: Ilyonectria, Fusarium, Tetracladium, Cladosporium, Rhizophagus, Alternaria, and Perisporiopsis (Table 2). Among them, Ilyonectria was the most dominant genera in the symptomatic samples, with an average abundance of 75.85 to $80.14 \%$. However, Ilyonectria was rarely detected in the asymptomatic samples. The abundance of Fusarium and the other genera in the symptomless samples were higher than that of the symptomatic samples, which was the opposite result found for Ilyonectria. These observations indicate that Ilyonectria might be the dominant pathogen inducing rusty root rot of $P$. notoginseng.

Diversity of pathogens and their pathogenicity. Candidate fungi were isolated from typical rusty root rot of $P$. notoginseng on PDA

Table 1. The fungal diversity of symptomatic and asymptomatic samples. PLRY2 and PLRY3 are asymptomatic root samples from 2- and 3-year-old sites, respectively; PLDRY2 and PLDRY3 are symptomatic root samples from 2- and 3-year-old sites, respectively

\begin{tabular}{lccccc}
\hline Sample & Coverage (\%) & OTUs $^{\mathbf{a}}$ & Chao1 $^{\mathbf{b}}$ & ACE $^{\mathbf{c}}$ & Shannon index $^{\mathbf{d}}$ \\
\hline PLRY2 & 99.32 & 229 & 467 & 768 & 2.75 \\
PLDRY2 & 99.49 & 181 & 296 & 311 & 1.32 \\
PLRY3 & 99.49 & 295 & 443 & 556 & 2.34 \\
PLDRY3 & 99.43 & 163 & 235 & 292 & 1.28 \\
\hline
\end{tabular}

${ }^{\text {a }}$ OTUs $=$ the classification of the object as the general term (species).

${ }^{\mathrm{b}} \mathrm{Chao} 1=$ the total number of species.

${ }^{c}$ Ace $=$ common indices of the estimated total species.

${ }^{\mathrm{d}}$ Shannon index $=$ diversity index.

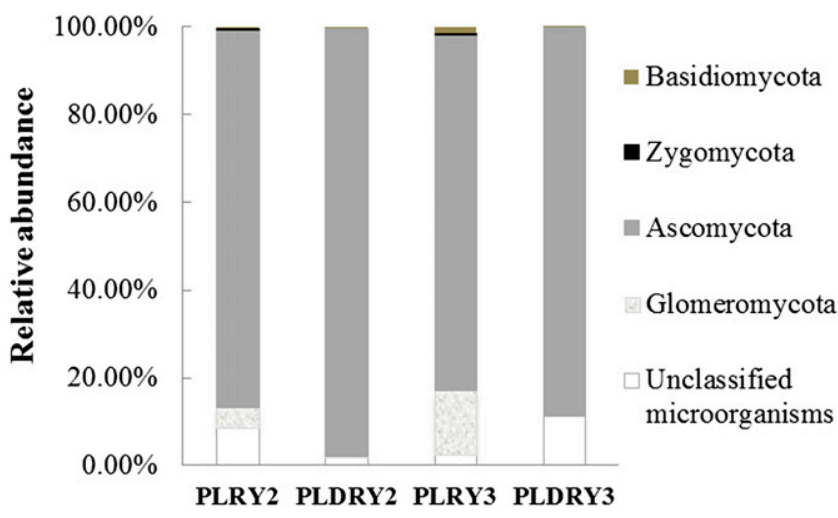

Fig. 1. Fungal community profiles and compositions based on Illumina Miseq data at the phylum level (PLRY2 and PLRY3 are asymptomatic root samples from 2- and 3-year-old sites, respectively; PLDRY2 and PLDRY3 are symptomatic root samples from 2- and 3-year-old sites, respectively). plates at $25^{\circ} \mathrm{C}$ for 14 days. Four morphologically different candidates (DRF1, G1A, G2B, and G3B) were continuously isolated, and G3Blike cultures were consistently obtained with the highest frequency. These candidates were accordingly employed to test their pathogenicity using in vitro and in vivo assays.

In the in vitro experiment, two of the four candidates (G3B and DRF1) caused rotting of all the root cutting pieces 5 to 7 days post inoculation (Fig. 2A), and they were further tested on 2-year-old plants of $P$. notoginseng. In the in vivo assay, the typical rusty rot symptoms were observed on the plants inoculated with G3B, including chlorotic leaves and brown, sunken, necrotic lesions both on the roots and stems (Fig. 2B1 and B3). However, candidate DRF1 induced the yellowing of one or two leaves and a slight rot on the surface of the roots (Fig. 2B2) while no symptoms were observed on the control plants. The same pathogens were reisolated from the corresponding inoculated plants, but no pathogens were isolated from the roots of the control plants. For G3B, spores and their germination were observed on the roots (Fig. 2B4). Similar results were obtained for the three replicate experiments.

Molecular identification was carried out to identify the candidates DRF1 and G3B. The ITS sequence from DRF1 showed 99\% similarity with that of Fusarium solani C02006 (JX524019.1) and thus was identified as $F$. solani. For G3B, the ITS sequence shared $99 \%$ similarity with that of Ilyonectria mors-panacis (Hildebr) Cabral \& Crous (CBS 120361). Furthermore, the sequence of the His3 gene had 99\% similarity with that of I. mors-panacis CBS 120361 . Both of the phylogenetic trees based on ITS (Fig. 3A) and on His3 (Fig. 3B) indicated that G3B clustered with I. mors-panacis, indicating that G3B is likely an isolate belonging to $I$. mors-panacis.

The morphology of candidate G3B is shown in Figure 4. After incubating for 12 days on PDA, the resulting colonies were cotton-like to felt-like in texture and orange-white to brownish gray with average diameters of $32.5 \mathrm{~mm}$. Macroconidia, cylindrical with both ends broadly rounded and 1-3-septate, formed predominantly on simple conidiophores. Microconidia that formed on complex conidiophores were ellipsoid to ovoid, and globose to subglobose chlamydospores were also produced.

Determination of the dominant pathogen based on qPCR. Four specific primers were designed, based on the sequences from Ilyonectria including G3B. Blastn and Megaline analyses revealed that the primers F2 and R2 had good specificity for I. mors-panacis. Experimental assays demonstrated that the primer pair F2-R2 gave amplification of an expectative single $249 \mathrm{bp}$ band from the DNA of the symptomatic roots, and the amplified sequences shared $100 \%$ similarity with the His 3 gene sequence of G3B. Therefore, F2-R2 was considered as an I. mors-panacis-specific primer pair which was employed in the following qPCR tests.

Altogether, 81 plants ( 48 symptomatic and 33 asymptomatic) were used to perform qPCR. All symptomatic roots had significantly higher relative quantities of I. mors-panacis than the corresponding asymptomatic roots (Fig. 5). For the samples collected in 2014, the symptomatic roots were sorted into three groups $(1,2,3)$ according to the symptoms (Supplementary Fig. S3). The groups with higher symptomatic grade showed more severe softening and browning. As shown in Fig. 5A and Supplementary Figure S3, the greater

Table 2. Relative abundance (\%) of dominant fungi between symptomatic (PLDRY2 and PLDRY3) and asymptomatic (PLRY2 and PLRY3) root samples

\begin{tabular}{lcccr}
\hline Genus & PLRY2 & PLDRY2 & PLRY3 & PLDRY3 \\
\hline Ilyonectria & 0.15 & 75.85 & 0.31 & 80.14 \\
Fusarium & 32.71 & 13.77 & 2.34 & 1.21 \\
Tetracladium & 12.33 & 0.12 & 59.12 & 1.09 \\
Cladosporium & 5.01 & 1.09 & 0.55 & 0.86 \\
Rhizophagus & 3.12 & 0.1 & 8.46 & 0.02 \\
Alternaria & 3.93 & 0.01 & 3.24 & 0.97 \\
Perisporiopsis & 5.36 & 0 & 0.01 & 0.04 \\
\hline
\end{tabular}


the severity of rusty rot symptoms, the significantly higher the relative quantity of $I$. mors-panacis that was determined. This indicates that the relative quantity of the pathogen correlated well with the symptom. Comparing the samples from different months during 2015 , all the rusty root rot samples showed high relative quantities of I. mors-panacis (Fig. 5B). For the same month, the pathogenic abundance of DR2 was always significantly different from DR3 $(P$ $<0.01)$. Except for the samples in June, it showed generally higher quantities in the 3-year-old sites than in the 2-year-old sites. These findings provide further evidence that I. mors-panacis is the dominant pathogen inducing rusty root rot of $P$. notoginseng.

\section{Discussion}

Recently, a worsening problem for $P$. notoginseng in Wenshan has been associated with a root rot apparently caused by many pathogens (Miao et al. 2006). In this study, molecular and culture-dependent methods were successfully applied to identify the dominant pathogen in rusty root rot of $P$. notoginseng.

Rapid identification of the dominant pathogen using multiple methods. The fungal community based on ITS pyrosequencing definitively demonstrated that Ilyonectria was the predominant pathogen causing rusty root rot of $P$. notoginseng. Fusarium is commonly isolated as a pathogen in many rotted plants (Chang et al. 2015); however, the abundance of Fusarium was much lower in the symptomatic roots $(1.21$ to $13.77 \%)$ than in the asymptomatic roots $(2.34$ to $32.71 \%$ ) of P. notoginseng. On the other hand, Ilyonectria dominated in the symptomatic roots with 75.85 to $80.14 \%$ abundance. These observations indicate that Fusarium might not be an important pathogen for $P$. notoginseng, or at least not a dominant pathogen, which differs from the findings of a previous report (Ma et al. 2013). However, the present observations of the dominant Ilyonectria and the minor presence of Fusarium in the symptomatic roots are consistent with the recent survey of fungal communities on symptomatic $P$. notoginseng (Wu et al. 2015). Therefore, fungal community analysis is an effective method for "overviewing" the possible dominant pathogen in the symptomatic samples, providing effective guidance for elucidating the specific important pathogen.

Using the above observations for guidance, we successfully and consistently obtained four fungal pathogenic candidates different morphologically from rusty root rot based on a modified culture method. However, using normal cultural methods, we previously failed to obtain Ilyonectria from rusty root rot of $P$. notoginseng many times over two years, and then mainly Fusarium was isolated. It is known that Fusarium can include around 300 distinct species (Aoki et al. 2014). They usually grow quickly and thus are easily cultured within 3 to 5 days (Ali et al. 2013), and can often inhibit the slow growth of other fungi such as Ilyonectria spp. (Aiello et al. 2013). It has been reported that the difficulty in studying C. destructans (Ilyonectria) is related in part to chlamydospores being the main form found in soil that rarely germinate, and when they do so, the mycelia grow slowly (Yoo et al. 1996). This explains well why Fusarium used to be deemed the pathogen of rusty root rot rather than Ilyonectria (Miao et al. 2006). Based on the fungal community guidance and the properties of Ilyonectria, we modified the culture method, including the use of higher dilutions of the samples ground with a mortar and pestle that were spread on PDA plates and delaying cultivation to 7 to 14 days at $25^{\circ} \mathrm{C}$. Eventually, a pathogen with the typical morphology of Ilyonectria, chestnut and felty mycelium with concentric zonation (Cabral et al. 2012), was consistently obtained from the symptomatic roots of $P$. notoginseng. This demonstrates that the single culture-dependent method is not a reliable way to discover pathogens, since most microbes are difficult or even impossible to culture. Fungal metagenomics, as well as a modified culture method, can compensate for the shortage of the traditional culture method.

Ilyonectria spp. were subsequently demonstrated, through both in vitro and in vivo experiments, to be the important pathogens causing serious symptoms similar to those observed in the field (Supplementary Fig. S1). Many more symptomatic samples were demonstrated to have Ilyonectria as the dominant pathogen through qPCR. Therefore,
A

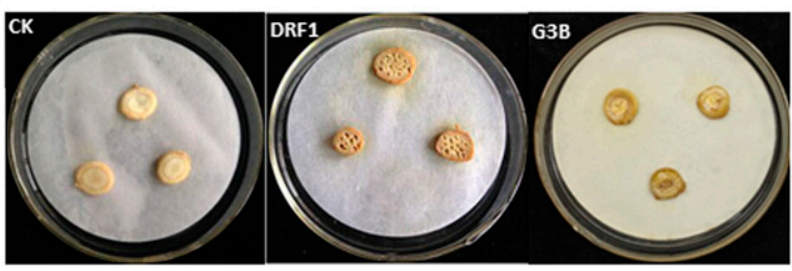

B1

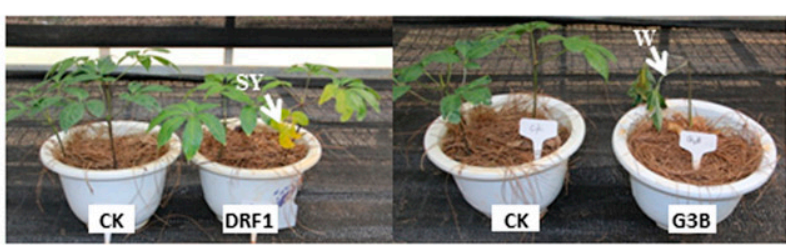

B2

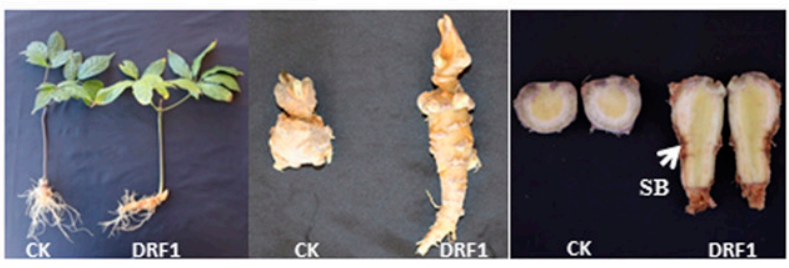

B3

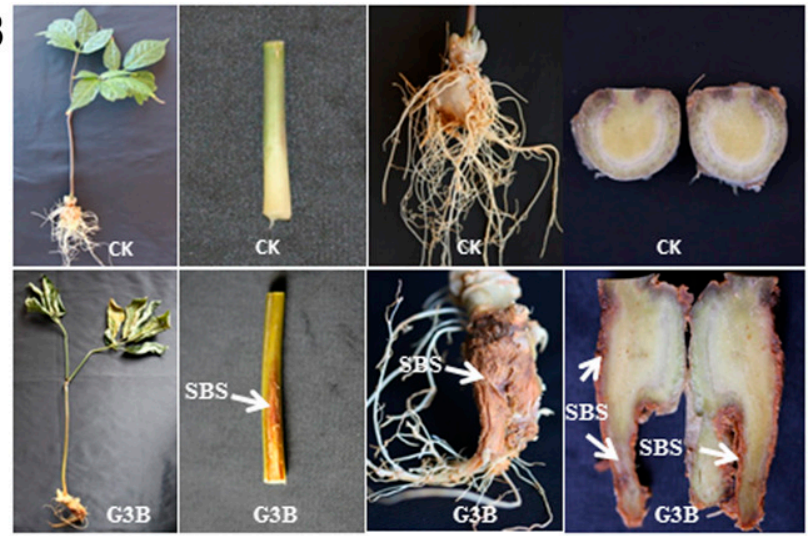

B4

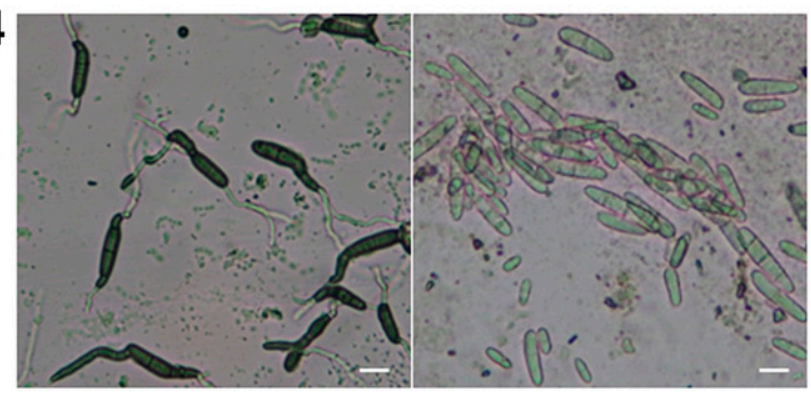

Fig. 2. In vitro, A, and in vivo, B, experiments of pathogenesis on root cutting species and plants for fungal isolates. A, symptoms caused by DRF1 and G3B on the root slices of $P$. Notoginseng. B1, symptoms caused by DRF1 and G3B on P. Notoginseng. Arrows showed slight yellowing (SY) for DRF1 and serious wither (W) for G3B. B2, details of the symptoms caused by DRF2 on the plants and the roots from B1. Arrow showed slight browning (SB) for the root by DRF2. B3, details of the symptoms caused by G3B on the plants and the roots from B1. Arrows showed severe browning and softening (SBS) of the stems and the roots by G3B. B4, details of the morphology of G3B observed on the roots from B1. CK: controls. Bars $=10 \mu \mathrm{m}$. 
multiple methods, including fungal metagenomics, modified cultural methods, pathogenesis, and qPCR are valuable combinational ways to identify the dominant pathogen.

Ilyonectria mors-panacis is the dominant pathogen inducing rusty root rot of $\boldsymbol{P}$. notoginseng. The major candidate G3B from the symptomatic roots, which caused similar typical symptoms, was identified as I. mors-panacis based both on morphological characteristics and on the ITS and His3 gene sequences. In 2014, Cylindrocarpon destructans var. destructans (I. radicicola) was first reported to cause the root rot of $P$. notoginseng (Mao et al. 2014). In fact, I. mors-panacis is one of the recently defined species from the complex of $C$. destructans $($ teleomorph $=$ Neonectria radicicola $)$ which underwent a complicated process of transformation as shown in the following.

The genus Cylindrocarpon includes species of Nectria-forming chlamydospores, with $C$. destructans being the most important member of this genus (Brayford 1993). Cylindrocarpon destructans, a species complex, is closely related to a number of well-known pathogenic

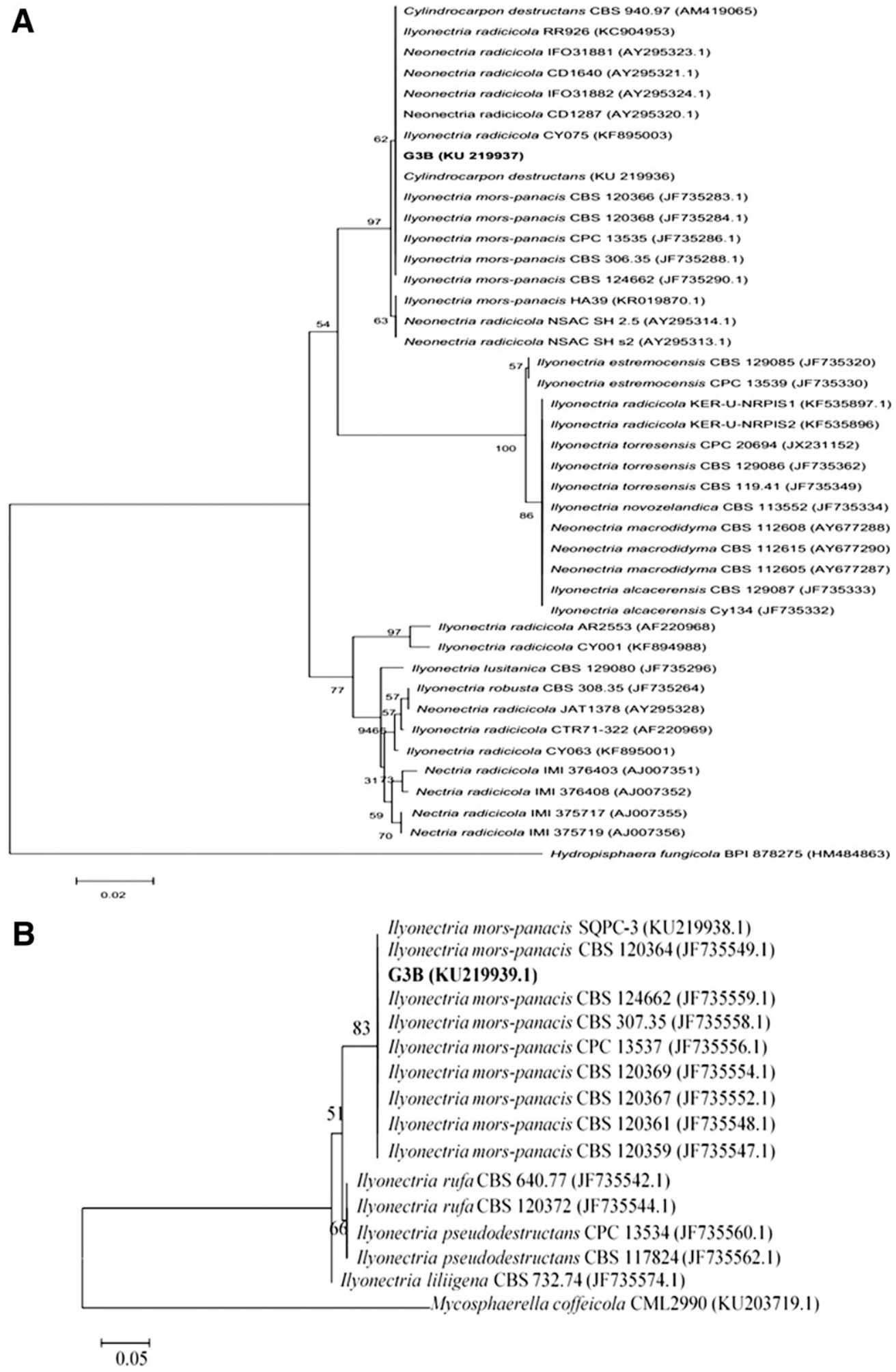

Fig. 3. Phylogenetic trees constructed by the neighbor-joining method based on ITS, A, and His3, B, gene sequences. Numbers on branches are the bootstrap values. GenBank accession numbers are given in parentheses. 
species in the family Nectriaceae, including other species of Cylindrocarpon, as well as Cylindrocladium (Rossman et al. 1999). Cylindrocarpon and Cylindrocarpon-like species have since been commonly associated with rot and decay of woody and herbaceous plants (Domsch et al. 2007), and have caused serious losses of ginseng (Panax quinquefolium) (Seifert et al. 2003), grapevines (Halleen et al. 2006), and apple (Tewoldemedhin et al. 2011)

Mantiri et al. (2001) estimated that there are approximately 125 described species of Cylindrocarpon. Most of the teleomorphs of Cylindrocarpon (groups 1, 2, and 4; Booth 1966) have been classified into the genus Neonectria Chaverri et al. (2011) based on phylogenetical and phenotypical characteristics, redefined Neonectria into four genera: anamorphs Ilyonectria (Booth's group 3) with chlamydospores; teleomorph genera Neonectria/Cylindrocarpon sensu stricto (groups 1 and 4); and Rugonectria and Thelonectria (group 2) without chlamydospores. Cabral et al. (2012) renamed the Nectria/Neonectria radicicola (including $C$. destructans) complex as Ilyonectria radicicola complex, and reclassified the fungi in this complex into 15 species under the genus Ilyonectria. In particular, highly virulent $I$. radicicola isolates of $P$. ginseng (IFO31881, IFO31882, NSAC SH1, and NSAC SH2.5) were included in I. mors-panacis (Song et al. 2014).

The present study demonstrates that the dominant pathogen G3B is phylogenetically and phenotypically similar to I. mors-panacis. Based on ITS sequences (Fig. 3A), G3B clustered well with the type strain of I. mors-panacis CBS 306.35 (JF735286.1), rot-causing $N$. radicicola IFO31881 (AY295323.1) on $P$. ginseng, and I. radicicola RR926 (KC904953) on P. notoginseng (Mao et al. 2014). Similar result was obtained based on His3 gene sequences (Fig. 3B). In addition, pathogen G3B had typical morphological characteristics similar to those of I. mors-panacis as well as chlamydospores (Chaverri et al. 2011). Therefore, it is determined that I. mors-panacis is the specific pathogen causing rusty root of $P$. notoginseng.

In conclusion, I. mors-panacis is the dominant pathogen responsible for inducing the severe rusty root rot of $P$. notoginseng. It is one

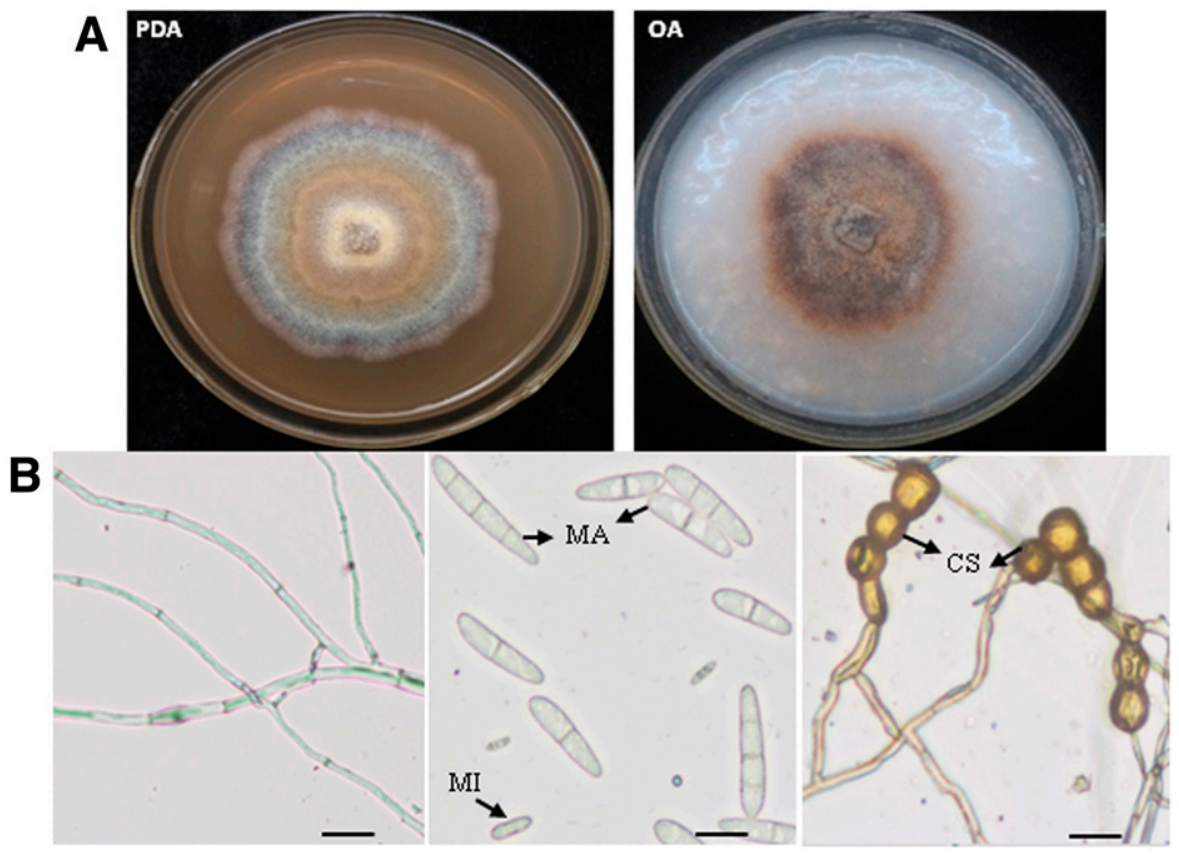

Fig. 4. Colony, A, and cell, B, morphologies of pathogen G3B on PDA or OA 12 days post cultivation at $25^{\circ} \mathrm{C}$. Bars, $10 \mu \mathrm{m} ; \mathrm{Ml}=$ microconidia; $\mathrm{MA}=$ macroconidia; $\mathrm{CS}=$ chlamydospores.

A

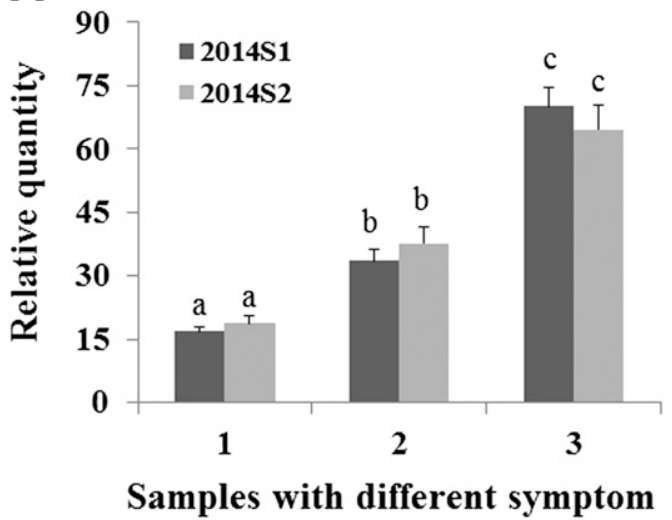

B

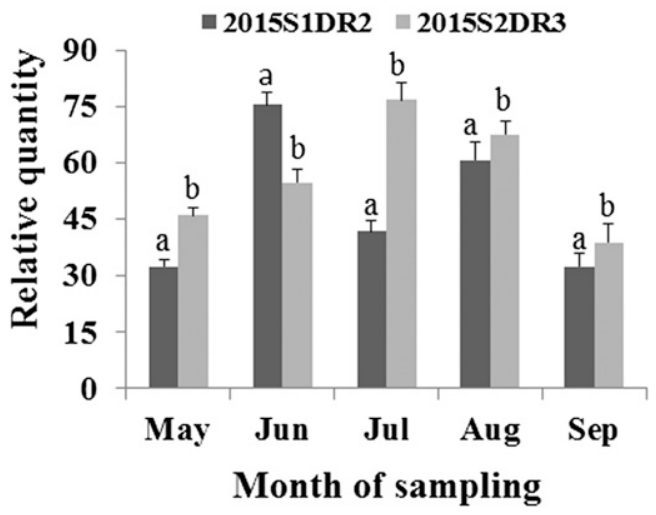

Fig. 5. qPCR confirmation of the dominant pathogen I. mors-panacis in the roots of $P$. notoginseng sampled during 2014 and 2015 . A, samples with different symptoms obtained from two different sites in 2014. While the symptoms were similar within each group between both sites, significant differences were found between the groups as shown by the different letters $(P<0.01)$. B, samples obtained from 2- (DR2) and 3-year-old (DR3) sites during different months in 2015. For each month, the pathogenic abundance of DR2 was significantly different from DR3 $(P<0.01)$, as shown by the different letters. 
of the causal organisms leading to $P$. notoginseng replanting problems, although other detrimental factors may also be involved. Understanding the genetic and pathogenic properties of I. morspanacis will be important for developing suitable root rot disease management strategies in the future.

\section{Acknowledgments}

This research is supported by the Zhejiang Province Public Agricultural Project (2015C32104), Zhejiang Natural Science Foundation of China (LY16C030002), Chinese National Natural Science Foundation (81673537), and key research and development projects in Zhejiang Province (2015C02030). We are grateful to Dr. Greg Duns for his valuable comments on the manuscript and to Sanchi Technology Co. Ltd. in the Wenshan region of Yunnan Province for all the materials.

\section{Literature Cited}

Abdel-Monaim, M. F., and Abo-Elyousr, K. A. M. 2012. Effect of preceding and intercropping crops on suppression of lentil damping-off and root rot disease in New Valley-Egypt. Crop Prot. 32:41-46.

Aiello, D., Guarnaccia, V., Vitale, A., Cirvilleri, G., Granata, G., Epifani, F., Perrone, G., Polizzi, G., Groenewald, J. Z., and Crous, P. W. 2013. Ilyonectria palmarum sp. nov. causing dry basal stem rot of Arecaceae. Eur. J. Plant Pathol. 138:347-359.

Ali, R., Khan, H., Ahmad, F., and Ahmad, N. 2013. Colony colour and texture of different isolates of Fusarium solani, the cause of root rot disease of Okra (Abelmoschus esculentusl) in Peshawar. Asian J. Agric. Biol. 1:190-193.

Aoki, T., O'Donnell, K., and Geiser, D. M. 2014. Systematics of key phytopathogenic Fusarium species: Current status and future challenges. J. Gen. Plant Pathol. 80: 189-201.

Booth, C. 1966. The genus Cylindrocarpon. Mycol. Pap. 104:1-54.

Brayford, D. 1993. Cylindrocarpon. Pages 103-106 in: Methods for Research on Soilborne Phytopathogenic Fungi. L. L. Singleton, J. D. Mihail, and M. Rush, eds. American Phytopathological Society, St. Paul, MN.

Cabral, A., Groenewald, J. Z., Rego, C., Oliveira, H., and Crous, P. W. 2012. Cylindrocarpon root rot: Multi-gene analysis reveals novel species within the Ilyonectria radicicola species complex. Mycol. Prog. 11:655-88.

Caporaso, J. G., Lauber, C. L., Walters, W. A., Berg-Lyons, D., Lozupone, C. A., Turnbaugh, P. J., Fierer, N., and Knight, R. 2011. Global patterns of 16S rRNA diversity at a depth of millions of sequences per sample. P. Natl. Acad. Sci. 108 (S1):4516-4522.

Cardenas, E., and Tiedje, J. M. 2008. New tools for discovering and characterizing microbial diversity. Curr. Opin. Biotechnol. 19:544-549.

Chang, K. F., Hwang, S. F., Conner, R. L., Ahmed, H. U., Zhou, Q., Turnbull, G. D., Strelkov, S. E., Mclaren, D. L., and Gossen, B. D. 2015. First report of Fusarium proliferatum causing root rot in soybean (Glycine max L.) in Canada. Crop Prot. 67:52-58.

Chaverri, P., Salgado, C., Hirooka, Y., Rossman, A. Y., and Samuels, G. J. 2011. Delimitation of Neonectria and Cylindrocarpon (Nectriaceae, Hypocreales, Ascomycota) and related genera with Cylindrocarpon-like anamorphs. Stud. Mycol. 68:57-78.

Chen, Y., Wang, Y., Feng, G., and Li, Z. 2001. Relationship between root rot of Panax notoginseng and ecological conditions. Yunnan Agric. Sci. Technol. 6:33-35

Crous, P. W., Groenewald, J. Z., Risede, J. M., Simoneau, P., and Hyde, K. D. 2006. Calonectria species and their cylindrocladium anamorphs: Species with clavate vesicles. Stud. Mycol. 55:213-226.

Domsch, K. H., Gams, W., and Anderson, T. H. 2007. Compendium of Soil Fungi, 2nd Ed. IHW-Verlag, Eching, Germany.

Glass, N. L., and Donaldson, G. C. 1995. Development of primer sets designed for use with PCR to amplify conserved genes from filamentous ascomycetes. Appl. Environ. Microbiol. 61:1323-1330.

Guan, H., Zhang, Y., Chen, Y., Yang, J., and Sun, S. 2011. On the connections between Sanchi root rot and edaphon dynamics in no-tillage sanchi-planted soil. Pages 1598-1602 in: 2011 International Conference on Computer Distributed Control and Intelligent Environmental Monitoring. IEEE, Piscataway, NJ

Guo, H. B., Cui, X. M., An, N., and Cai, G. P. 2010. Sanchi ginseng (Panax notoginseng (Burkill) FH Chen) in China: Distribution, cultivation, and variations. Genet. Resour. Crop Evol. 57:453-460.

Halleen, F., Fourie, P. H., and Crous, P. W. 2006. A review of black foot disease of grapevine. Phytopathol. Mediterr. 45:55-67.

Heydari, A., Khodakaramian, G., and Zafari, D. 2014. Occurrence, genetic diversity and pathogenicity characteristics of Pseudomonas viridiflava inducing alfalfa bacterial wilt and crown root rot disease in Iran. Eur. J. Plant Pathol. 139:299-307.

Hong, J., Hu, J. Y., Liu, J. H., Zhou, Z., and Zhao, A. F. 2014. In vitro antioxidant and antimicrobial activities of flavonoids from Panax notoginseng flowers. Nat. Prod. Res. 28:1260-1266.
Hong, S. H., Bunge, J., Jeon, S. O., and Epstein, S. S. 2006. Predicting microbial species richness. Proc. Natl. Acad. Sci. USA 103:117-122.

Hu, X. F., Ying, X., He, Y. B., Gao, Y. Y., Chen, H. M., and Chen, J. S. 2008 Characterization of Pectobacterium carotovorum subsp. carotovorum causing soft-rot disease on Pinellia ternata in China. Eur. J. Plant Pathol. 120:305-310.

Latha, P., Anand, T., Prakasam, V., Jonathan, E. I., Paramathma, M., and Samiyappan, R. 2011. Combining Pseudomonas, Bacillus, and Trichoderma strains with organic amendments and micronutrient to enhance suppression of collar and root rot disease in physic nut. Appl. Soil Ecol. 49:215-223.

Li, X., Wang, G., Sun, J., Hao, H., Xiong, Y., Yan, B., Zheng, Y., and Sheng, L. 2007. Pharmacokinetic and absolute bioavailability study of total Panax notoginsenoside, a typical multiple constituent traditional Chinese medicine (TCM) in rats. Biol. Pharm. Bull. 30:847-851.

Lu, X. H., Jiao, X. L., Chen, A. J., Luo, Y., and Gao, W. W. 2015. First report of Ilyonectria robusta causing rusty root of Asian ginseng in China. Plant Dis. 99: 156.

Ma, L., Cao, Y. H., Cheng, M. H., Huang, Y., Mo, M. H., Wang, Y., Yang, J. Z., and Yang, F. X. 2013. Phylogenetic diversity of bacterial endophytes of Panax notoginseng with antagonistic characteristics towards pathogens of root-rot disease complex. Anton. Leeuw. Int. J. G. 103:299-312.

Mantiri, F. R., Samuels, G. J., Rahe, J. E., and Honda, B. M. 2001. Phylogenetic relationships in Neonectria species having Cylindrocarpon anamorphs inferred from mitochondrial ribosomal DNA sequences. Can. J. Bot. 79:334-340.

Mao, Z. S., Long, Y. J., Zhu, Y. Y., Zhu, S. S., He, X. H., and Chen, Z. J. 2014 First report of Cylindrocarpon destructans var. destructans causing black root rot of sanqi (Panax notoginseng) in China. Plant Dis. 98:162.

Miao, Z. Q., Li, S. D., Liu, X. Z., Chen, Y. J., Li, Y. H., Wang, Y., Guo, R. J., Xia, Z. Y., and Zhang, K. Q. 2006. The causal microorganisms of Panax notoginseng root rot disease. Sci. Agric. Sin. 39:1371-1378.

Qian, P., Mi, C. Y., Jiang, K., Yang, R. H., Li, O., and Hu, X. F. 2016 Phylogenetic and metabolic responses of rhizosphere microbes to the cultivation of Panax notoginseng. J. Biobased Mater. Bio. 10: 370-377.

Reis, P., Cabral, A., Nascimento, T., Oliveira, H., and Rego, C. 2013. Diversity of Ilyonectria species in a young vineyard affected by black foot disease. Phytopathol. Mediterr. 52:335-346.

Rossman, A. Y., Samuels, G. J., Rogerson, C. T., and Lowen, R. 1999. Genera of Bionectriaceae, Hypocreaceae and Nectriaceae (Hypocreales, Ascomycetes). Stud. Mycol. 42:1-248

Scherm, B., Balmas, V., Spanu, F., Pani, G., Delogu, G., Pasquali, M., and Quirico, M. 2013. Fusarium culmorum: Causal agent of foot and root rot and head blight on wheat. Mol. Plant Pathol. 14:323-341.

Schloss, P. D., Westcott, S. L., Ryabin, T., Hall, J. R., Hartmann, M., Hollister, E. B., and Weber, C. F. 2009. Introducing mothur: Open-source, platformindependent, community-supported software for describing and comparing microbial communities. Appl. Environ. Microbiol. 75:7537-7541.

Seifert, K. A., McMullen, C. R., Yee, D., Reeleder, R. D., and Dobinson, K. F. 2003. Molecular differentiation and detection of ginseng-adapted isolates of the root rot fungus Cylindrocarpon destructans. Phytopathology 93: $1533-1542$.

Shishido, M. 2014. Black root rot caused by Diaporthe sclerotioides threatens cucurbit cultivation in Japan. Adv. Hortic. Sci. 28:208-213.

Song, J. Y., Seo, M. W., Kim, S. I., Nam, M. H., Lim, H. S., and Kim, H. G. 2014 Genetic diversity and pathogenicity of Cylindrocarpon destructans isolates obtained from Korean Panax ginseng. Mycobiol. 42:174-180.

Sun, Y., Ke, J., Ma, N., Chen, Z., Wang, C., and Cui, X. 2004. Effects of root rot on saponin content in Panax notoginseng. J. Chin. Med. Mater. 27:79-80.

Tewoldemedhin, Y. T., Mazzola, M., Mostert, L., and McLeod, A. 2011. Cylindrocarpon species associated with apple tree roots in South Africa and their quantification using real-time PCR. Eur. J. Plant Pathol. 129:637-651.

Tringe, S. G., and Hugenholtz, P. 2008. A renaissance for the pioneering $16 \mathrm{~S}$ rRNA gene. Curr. Opin. Microbiol. 11:442-446.

Wu, Z., Hao, Z., Zeng, Y., Guo, L., Huang, L., and Chen, B. 2015. Molecular characterization of microbial communities in the rhizosphere soils and roots of diseased and healthy Panax notoginseng. Anton. Leeuw. 108: 1059-1074.

Xia, P. G., Guo, H. B., Zhang, Y., Deyholos, M. K., Peng, L., Jia, Y. Y., Yan, X. J., Liu, Y., and Liang, Z. S. 2016. Wild Panax vietnamensis and Panax stipuleanatus markedly increase the genetic diversity of Panax notoginseng (Araliaceae) revealed by start codon targeted (SCoT) markers and ITS DNA barcode. Biochem. Syst. Ecol. 66:37-42.

Yoo, S. J., Cho, J. W., Jo, J. S., and Yu, S. H. 1996. Effect of physical and chemical factors on the formation and germination of chlamydospore of Cylindrocarpon destructans causing root rot of Panax ginseng. Korean J. Plant Pathol. 12: $422-7$

Yu, J., Zhou, X. F., Yang, S. J., Liu, W. H., and Hu, X. F. 2013. Design and application of specific $16 \mathrm{~S}$ rDNA-targeted primers for assessing endophytic diversity in Dendrobium officinale using nested PCR-DGGE. Appl. Microbiol. Biotechnol. 97:9825-9836. 